\title{
Study of High Accuracy Displacement Field Measurement by Digital Image Correlation
}

\author{
Yunxiang Wang ${ }^{1}$, Sujuan $\mathrm{Shi}^{1}$, Hao Zhou ${ }^{2}$, Daqing $\mathrm{Chen}^{2}$ and Hongping Huang ${ }^{1}$ \\ ${ }^{1}$ suzhou Institute of Measurement and Testing Technology' Suzhou, 215006, China \\ ${ }^{2}$ Physics Department, Soochow University' Suzhou, 215006, China \\ ${ }^{*}$ Corresponding author
}

\begin{abstract}
A new approach of high accuracy displacement field measurement in long-distance by digital image correlation is proposed. The long-distance measurement accuracy attained 1 micron with digital image correlation technique of 0.001 pixel bilinear interpolation. Integrated software package is exploited with language of $\mathrm{c}++$ and Matlab under Windows system . It combined with optics hardware to constitute a multi-function instrument for displacement field measurement. This system have features of high accuracy, real-time and non-contact measurement with computer control and is especially effective in flexivity measurement of bridge and cloverleaf junction. It also fit for dynamic displacement field measurement when a high speed image card was used. This technology extend digital image correlation method's application field.
\end{abstract}

Keywords-digital image correlation; flexibility ; displacement field; interpolation

\section{INTRODUCTION}

The measurement for displacement field in long-distance has been widely applied in the loading test on bridges and dynamic monitoring for large structure etc. Approaches such as testing by electronic total station and dial indicator are proposed in the above mentioned applications [1,2]. However, electronic total station with high accuracy leads to high-cost and the dial indicator requires high stability affecting the experimental results. It is important to develop non-contact measurement technique for displacement field in long-distance.

In this paper, a modern optical test technique with high precision is proposed based on optical imaging and digital image correlation. The natural or artificial random spots on the objects' surface are treated as the information carrier in which deformation information can be extracted. An experiment for the measurement of deflection on the cantilever has been carried out from remote distance. The accuracy of displacement field measurement can be up to 0.01 pixels which is 1 microns accuracy for the measurement distance 1 meter. The data transmission and interface processing program is also designed so that high-speed data acquisition, storage and processing can be easily achieved. Mesh and contour map of the displacement field could also be obtained conveniently by Matlab software which enables displacement field measurement for arbitrary point at real time.

\section{PRINCIPLES}

Digital image correlation measurement method has the superiority of high accuracy, real-time and non-contact. It is a important component of modern optical measurement method. It is assumed that there is a one - to - one correspondence between the reflective intensity distribution of the object $I(x, y)$ and the object's surface when the calculation of digital image correlation is taken (Figure (1)). Thus, the deformation of the object could be obtained by measuring the variance of $I(x, y)$. The displacement at the center of each sub-region $\mathrm{u}, \mathrm{v}$ in $\mathrm{x}$ and $\mathrm{y}$ direction separately can be calculated by the correlation searching algorithm for corresponding subregions before and after deformation. The formula for correlation is given by:

$$
C(u, v)=\frac{\sum_{i=1}^{m} \sum_{j=1}^{m}\left[I_{1}\left(x_{i}, y_{i}\right)-\overline{I_{1}}\right]\left[I_{2}\left(x_{i}+u, y_{i}+v\right)-\overline{I_{2}}\right]}{\sqrt{\sum_{i=1}^{m} \sum_{j=1}^{m}\left[I_{1}\left(x_{i}, y_{i}\right)-\overline{I_{1}}\right]^{2}} \sqrt{\sum_{i=1}^{m} \sum_{j=1}^{m}\left[I_{2}\left(x_{i}+u, y_{i}+v\right)-I_{2}\right]^{2}}}
$$

In Eq. (1), $I_{1}\left(x_{i}, y_{i}\right)$ and $I_{2}\left(x_{i}+u, y_{i}+v\right)$ are the gray-scale functions of the images before and after deformation; $\bar{I}_{1}$ and $\bar{I}_{2}$ are the averages of $I_{1}\left(x_{i}, y_{i}\right)$ and $I_{2}\left(x_{i}+u, y_{i}+v\right) ; C(u, v)$ is the cross correlation coefficient.

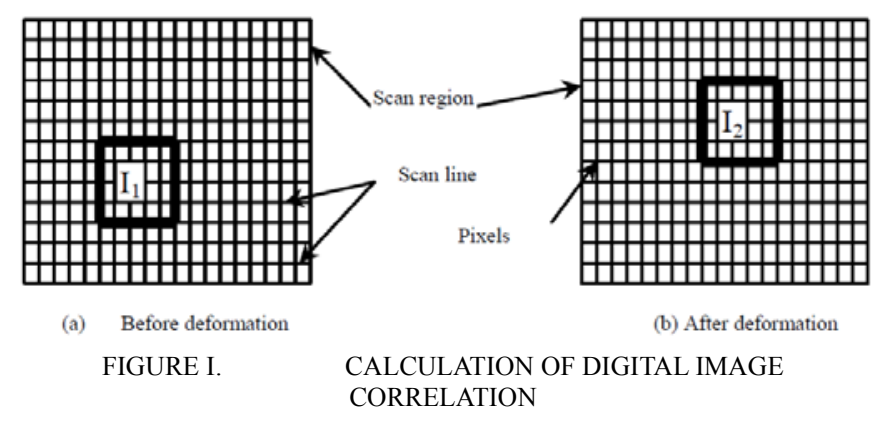

It requires sub-pixels extraction by interpolation approach when the digital image correlation calculation for the images obtained by the CCD is executed. A "coarse to fine" pixelsearching method is used in this paper: the displacement test is firstly calculated on integer pixels thus the correlation coefficients of the sub-image before and after deformation 
could be obtained. The displacement $(\mathrm{u}, \mathrm{v})$ with maximum value of the correlation coefficients is the first approximation of the real displacement. Repeat this step until the required accuracy is achieved. In order to enhance the measurement precision, a fractional pixel which would be obtained by interpolation approach is added on the each original approximation.

\section{DigITAL IMAGE CORRELATION SYSTEM FOR DISPLACEMENT MEASUREMENT}

\section{A. Hardware}

The hardware of digital image correlation system is shown in Figure 2.The CCD has $768 \times 576$ pixels and a zoom camera lens $(\mathrm{f}=70-210 \mathrm{~mm})$ has been settled on the CCD to obtain the focused image of the object. The optical signal is converted into electronic signal by CCD. A digitalized gray image can be stored in the computer's memory by A/D transformation in image card $(758 \times 576$ pixel $\times 8$ bit, frame rate $25 \mathrm{f} / \mathrm{s})$.Define abbreviations and acronyms the first time they are used in the text, even after they have been defined in the abstract. Abbreviations such as IEEE, SI, MKS, CGS, sc, dc, and rms do not have to be defined. Do not use abbreviations in the title or heads unless they are unavoidable.
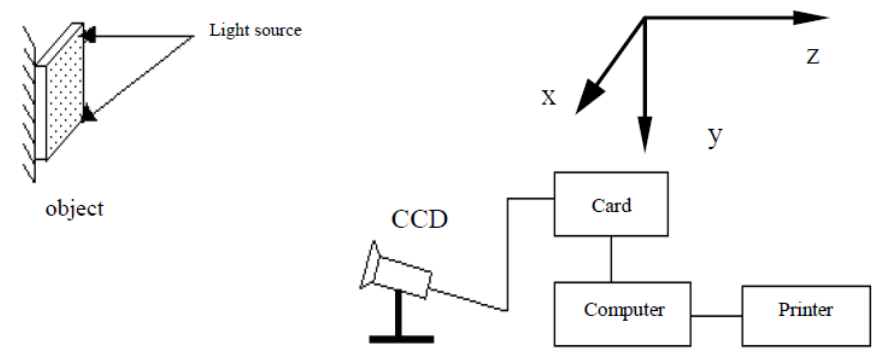

FIGURE II.

HARDWARE OF DIGITAL IMAGE CORRELATION MEASUREMENT SYSTEM.

\section{B. Software}

The digital image correlation calculation software plays the core role of the system. An integrated correlation measurement software is designed with many functions and instrumentation is attained. The automatic measurement program has been developed by using $\mathrm{C}+\mathrm{Builder}$ and Matlab. The bilinear interpolation method with 0.001 pixel precision is used in the correlation calculation.

The measurement process is shown in Figure 3. Since the noise of the imaging system would affect the correlation accuracy, it is essential to adjust the parameters of the optical system to increase contrasts and decrease noise of the image. In our experiment, the random noise of the image is controlled under 3 gray levels to achieve higher precision.

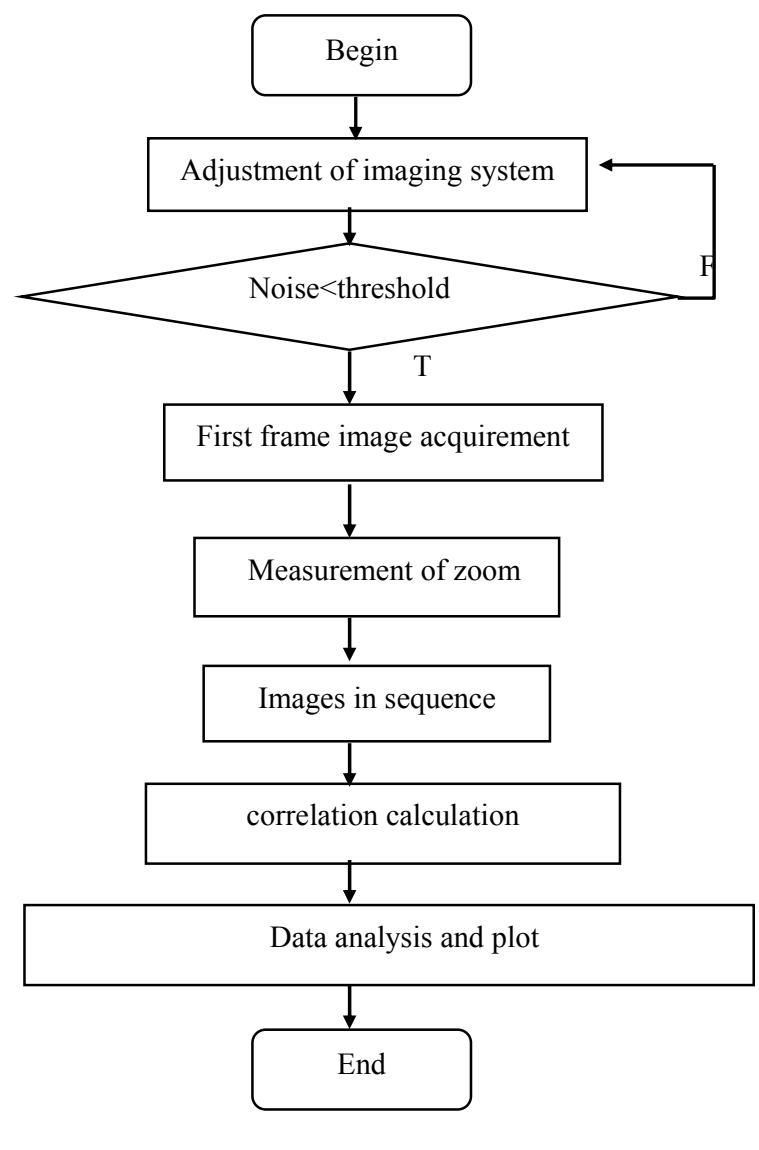

FIGURE URE III. THE MEASUREMENT PROCESS

\section{EXPERIMENT}

\section{A. Measurement of Deflection on the Cantilever}

The structure of the cantilever is shown in Figure 4. It is under pressure of concentrated load P. The deflection can be expressed as:

$$
f=\frac{P}{6 E I} y^{2}(3 l-y) .
$$

Eq. (2) can be rewritten as:

$$
f_{\text {max }}=\frac{P}{6 E I} l^{2}(3 l-l),
$$

in which y has the maximum value. The result of Eq.(3) is fed into Eq.(2),we can deduce that:

$$
f=\frac{1}{2}\left(\frac{y}{l}\right)^{2}\left(3-\frac{y}{l}\right) f_{\max } .
$$

In Eq.(4), $f_{\max }$ is the deflection at $y=l$ which can be measured by dial indicator. The result by dial indicator is calculated in Eq.(4) as the theoretical value compared with the value obtained by digital image correlation method. 


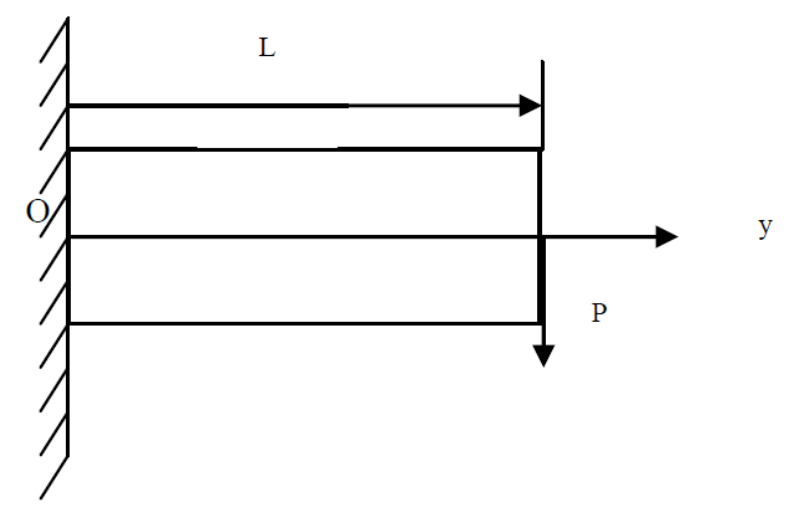

FIGURE IV,

CONCENTRATED LOAD P ON THE CANTILEVER

Table 1 shows the measurement result by digital image correlation method:

TABLE I.

MEASUREMENT OF DEFLECTION ON THE CANTILEVER

\begin{tabular}{|c|c|c|c|c|c|c|}
\hline $\mathbf{y} / \mathbf{c m}$ & 0.277 & 0.554 & 0.831 & 1.11 & 1.386 & 1.66 \\
\hline $\begin{array}{c}\text { MEASURMENT } \\
\text { VALUE } / \boldsymbol{\mu m}\end{array}$ & 1.042 & 5.21 & 9.38 & 15.63 & 23.96 & 32.29 \\
\hline $\begin{array}{c}\text { THEORETICAL } \\
\text { VALUE } / \mu \mathrm{m}\end{array}$ & 0.98 & 4.96 & 9.13 & 15.75 & 22.96 & 31.83 \\
\hline $\mathbf{E R R O R / \mu \mathrm { m }}$ & 0.062 & 0.25 & 0.25 & 0.12 & 1.00 & 0.46 \\
\hline $\mathbf{y} / \mathbf{c m}$ & 1.94 & 2.22 & 2.49 & 2.77 & 3.05 & \\
\hline $\begin{array}{c}\text { MEASURMENT } \\
\text { VALUE } / \boldsymbol{\mu m}\end{array}$ & 42.71 & 54.21 & 66.63 & 80.87 & 96.84 & \\
\hline $\begin{array}{c}\text { THEORETICAL } \\
\text { VALUE } / \boldsymbol{\mu m}\end{array}$ & 41.83 & 53.83 & 67.13 & 81.63 & 97.28 & \\
\hline ERROR/ $\boldsymbol{\mu m}$ & 0.88 & 0.38 & 0.5 & 0.76 & 0.44 & \\
\hline
\end{tabular}

In Table 1, the error between measurement and theoretical value is all under $1 \mathrm{um}$ which means the measurement accuracy reach 0.01 pixel.

\section{B. Measurement of Displacement Field on the Cantilever}

Figure 5 is the surface map and contour map of displacement of the cantilever in (a), (b), (c) and (d).The results indicate consistency with the elastic mechanical theory.
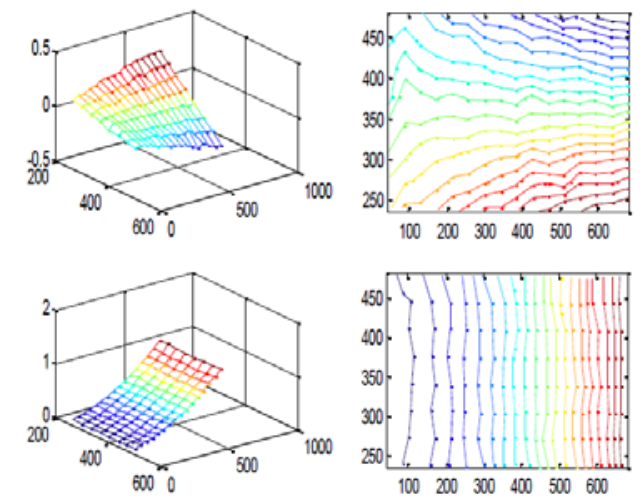

FIGURE V.

DISPLACEMENT FIELD OF CANTILEVER UNDER PRESSURE OF CONCENTRATED LOAD P

\section{CONCLUSIONS}

In this paper, we propose a displacement field measurement technique with high precision from remote distance. It is based on the digital image correlation method. An integrated correlation measurement program with many functions is designed and instrumentation is attained. The accuracy of displacement field measurement can be up to 0.01 pixels under the interpolation with 0.001 pixel precision. This technique has the superiority of real-time and non-contact which is adapt to the measurement for dynamic or static load characteristics of the bridge or expressway. With the development of this technique, it could be an important tool for displacement measurement.

\section{REFERENCES}

[1] Yuan Ruiping,Li Zhu. Loading test research on bridges in yinghuang project[J]. Journal of Shijiazhuang Railway Institute, 2002,15(B08):6870 (in chinese).

[2] Gou Jinjun, Xu Liang,Jiang Jianjing. Large Structure Dynamic Monitoring Using Gps Technology[J]. Journal of East China Geological Institute, 2002,25(4):324-332 (in chinese) .

[3] Jin Guanchanga, Wu Zhena, Bao Nikengb, Yao Xuefeng. Digital speckle correlation method with compensation technique for strain field measurements [J]. Optics and Lasers in Engineering, 2003,39:457-464.

[4] Ma Shaopeng, Jin Guanchang, Pan Yishan. Study on the White Light DSCM Method for Deformation Measurement of Rock Materials [J]. Journal of Experimental Mechnacis, 2002,17(1):10-16 (in chinese ).

[5] Gong Haohan, Jiang Jinhu, Chen Daqing. Displacement Measurement by Grid Digital Image Correlation[J]. Journal of Experimental Mechnacis, 2000,15(2):246-252 (in chinese).

[6] Chen Siying, Huang Chenfuang,Duan Zhuping,Digital speckle correlation method and its applicationon impact deformation measurement[J]. Chinese journal of lasers, 2004,31(6) : 735-739(in chinese).

[7] Peters.W.H.,Ranson.W.F., Digital imaging techniques in experimental stress analysis[J]. Opt.Eng., 1982,21(5): 427-431.

[8] A.Asundi \& H.North. White-light Speckle Method-Current Trends [J]. Optics and Lasers in Engineering, 1998,29:159-169.

[9] Rui Jiabai, Jin Guanchang,Xu Bingye . A New Digital Speckle Correlati0n Method and Its Application[J] . Acta Mechnaca Sinica, 1994,26(5): 599-607.(in chinese).

[10] Gao Jianxin, Zhou Xingeng. Resolution Analysis of Digital Image Measurement System[J]. Acta Mechnaca Solida Sinica, 1997.18(2): 163-166(in chinese). 